\title{
Perfect Matchings in Planar Cubic Graphs
}

\author{
Maria Chudnovsky ${ }^{1}$ \\ Columbia University, New York, NY 10027 \\ Paul Seymour ${ }^{2}$ \\ Princeton University, Princeton, NJ 08544
}

January 24, 2008; revised July 16, 2009

${ }^{1}$ This research was conducted while the author served as a Clay Mathematics Institute Research Fellow.

${ }^{2}$ Supported by ONR grant N00014-01-1-0608 and NSF grant DMS-0070912. 


\begin{abstract}
A well-known conjecture of Lovász and Plummer from the mid-1970's, still open, asserts that for every cubic graph $G$ with no cutedge, the number of perfect matchings in $G$ is exponential in $|V(G)|$. In this paper we prove the conjecture for planar graphs; we prove that if $G$ is a planar cubic graph with no cutedge, then $G$ has at least

$$
2^{|V(G)| / 655978752}
$$

perfect matchings.
\end{abstract}




\section{Introduction}

In 1998, Schrijver [4] proved the "Schrijver-Valiant conjecture", a lower bound on the number of perfect matchings in a $k$-regular bipartite graph. A consequence of this is that every cubic bipartite graph has exponentially many perfect matchings.

But what about non-bipartite cubic graphs? They need not have any perfect matchings at all, so let us confine ourselves to cubic graphs without cutedges. In that case, Lovász and Plummer [3] conjectured in the mid-1970's that again such a graph $G$ must have exponentially many perfect matchings. This has proved to be a challenging question, and is still open; and the best lower bound currently known is $|V(G)| / 2+1$ (except for one graph with twelve vertices and only six perfect matchings), proved recently by Kral, Sereni and Stiebitz [2]. In this paper we prove the conjecture for planar cubic graphs, the following:

\subsection{Let $G$ be a planar cubic graph with no cutedge. Then $G$ has at least}

$$
2^{|V(G)| / 655978752}
$$

\section{perfect matchings.}

Planarity provides two advantages. First, we have the four-colour theorem, which tells us that planar cubic graphs without cutedges are 3-edge-colourable, and thus provides us with a way to produce triples of perfect matchings that cover all the edges. (However, it is not true that these graphs necessarily have exponentially many 3-edge-colourings - unlike bipartite cubic graphs, which do so we need to use the four-colour theorem in an indirect way.) The second advantage of planarity is that we have regions, a source of cycles of bounded length whose deletion does not reduce the connectivity of the graph very much; and these will be key to the proof.

The proof breaks into two parts; first we prove the statement for cyclically 4-edge-connected planar cubic graphs, and then we deduce it for general (planar) cubic graphs.

The idea in the cyclically 4-edge-connected case is, we first find linearly many even length cycles, disjoint and carefully chosen (ideally we would like them far apart, and each bounding either one region or the union of two regions). This set of cycles has exponentially many subsets; let $X$ be one

of its subsets. For each cycle in $X$, delete its even edges and replace its odd edges by pairs of parallel edges. (Let us call this "flipping" the cycle.) We arrange that for each $X$ the graph we produce will be a planar cubic graph with no cutedges, and will therefore be 3-edge-colourable, because of the four-colour theorem. Consequently there will be a triple of perfect matchings of the original graph, such that we can reconstruct $X$ from this triple. This shows that the original graph had exponentially many triples of perfect matchings, and therefore exponentially many perfect matchings. The problem here is that we cannot necessarily find such a large set of even cycles sufficiently far apart for them all to be flippable independently without producing cutedges, and we sometimes have to make do with something a little less (we make use of the fact that there are two ways to flip a cycle, depending on which we designate as its "even" edges, and we only need one of the two ways to work).

The second half of the proof, when the graph is not cyclically 4-edge-connected, is quite nontrivial, rather surprisingly so, and it is the proof for this case that makes the constant in our main result so large. We find it necessary to retain the stronger statement proved in the cyclically 4-edgeconnected case, that we have linearly many disjoint cycles that can all be independently "flipped", rather than just that there are exponentially many perfect matchings. 


\section{Good looks, bracelets, and perfect matchings}

Let us be more precise. Graphs in this paper are all finite and loopless, but not necessarily simple (they may have parallel edges). For a graph $G$, if $X \subseteq V(G)$ we define $\delta_{G}(X)=\delta(X)$ to be the set of edges of $G$ with one end in $X$ and one end in $V(G) \backslash X$; and if $v \in V(G)$ we write $\delta(v)$ for $\delta(\{v\})$. A cut in $G$ is a set of edges $D$ such that $D=\delta(X)$ for some $X \subseteq V(G)$. If $w: E(G) \rightarrow\{-1,0,1\}$ and $Y \subseteq E(G)$, we define $w(Y)$ to be the sum of $w(e)$ for all $e \in Y$. If $G$ is cubic and $w: E(G) \rightarrow\{-1,0,1\}$ satisfies $w(\delta(v))=0$ for each $v \in V(G)$, we call $w$ a look of $G$. A look $w$ is said to be good if for every cut $D, w(D) \neq 1-|D|$. (In other words, if we delete from $G$ all the edges $e$ with $w(e)=-1$, and $f$ is a cutedge of the graph we produce, then $w(f)=1$.)

We observe first that:

2.1 Let $G$ be a planar cubic graph with $k$ good looks. Then $G$ has at least $k^{\frac{1}{3}}$ perfect matchings.

Proof. Let $w$ be a good look of $G$. Let $H$ be obtained from $G$ by deleting all edges $e$ with $w(e)=-1$, and adding an edge parallel to every edge $e$ with $w(e)=1$. Then $H$ is also planar and cubic, and since $w$ is a good look of $G$ it follows that $H$ has no cutedge. By the four-colour theorem, there are three perfect matchings in $H$ such that every edge of $H$ is in exactly one of them. Consequently there are three perfect matchings in $G$, say $F_{1}, F_{2}, F_{3}$, such that every edge $e$ is in $1+w(e)$ of them. In particular, $w$ can be reconstructed from a knowledge of $F_{1}, F_{2}, F_{3}$; and so no two different good looks produce the same triple $\left(F_{1}, F_{2}, F_{3}\right)$. We deduce that there are at least $k$ distinct triples of perfect matchings, and the theorem follows. This proves 2.1.

Let $C$ be a cycle of $G$ with even length, and let $w_{0}: E(C) \rightarrow\{1,-1\}$, such that the edges of $C$ are mapped alternately to 1 and to -1 . We call $w_{0}$ a bracelet on $C$. Any such map $w_{0}$ that arises in this way from some cycle $C$ is called a bracelet of $G$, and $C$ is its supporting cycle. Define $w: E(G) \rightarrow\{-1,0,1\}$ by $w(e)=w_{0}(e)$ if $e \in E(C)$, and $w(e)=0$ otherwise. Then $w$ is a look, and we call $w$ the look of the bracelet $w_{0}$. Any function $w: E(G) \rightarrow\{-1,0,1\}$ that arises in this way from some bracelet $w_{0}$ we call a bracelet look of $G$. (It is convenient for us to distinguish between a bracelet and its look, especially when we come to the later parts of the proof, because sometimes the same bracelet will occur in several different graphs.)

Again, let $G$ be cubic. A jewel-box for $G$ is a set $\mathcal{B}$ of bracelets of $G$, satisfying:

- every two members of $\mathcal{B}$ have disjoint supporting cycles

- for every subset $W \subseteq \mathcal{B}$, the sum of the looks of the members of $W$ is a good look.

We define $\beta(G)$ to be the cardinality of the largest jewel-box in $G$. Our main result is:

2.2 For every planar cubic graph $G$ with no cutedge, $\beta(G) \geq|V(G)| / 218659584$.

This implies that $G$ has at least $2^{|V(G)| / 218659584}$ good looks, and hence at least $2^{|V(G)| / 655978752}$ perfect matchings, by 2.1 ; so 1.1 follows from 2.2 .

\section{The cyclically 4-edge-connected case}

Let us say a cubic graph $G$ is cyclically 4-edge-connected or $C 4 C$ if it is 3-connected (and hence is simple and has at least four vertices) and for every set $X \subseteq V(G)$ with $|X|,|V(G) \backslash X|>1$ there are 
at least four edges between $X$ and $V(G) \backslash X$. Our goal in this section is to prove 2.2 (with a better constant) for $\mathrm{C} 4 \mathrm{C}$ planar cubic graphs. We shall show the following:

\subsection{For every $C 4 C$ planar cubic graph $G, \beta(G) \geq|V(G)| / 30976$.}

We first need a lemma. (To clarify - the degree of a vertex is the number of edges incident with it; a cycle has no repeated vertices; and the length of a path or cycle is the number of edges in it.)

3.2 Let $G$ be a simple planar graph, let $A \subseteq V(G)$ be stable, let $d>0$ be an integer, and let each member of $A$ have degree at most d in $G$. Then there exist $X \subseteq A$ and $Y \subseteq V(G) \backslash A$, such that

- $|X| \geq|A| /(64 d+8)$

- each member of $X$ is adjacent to at most two members of $Y$

- every path in $G$ of length at most three between two members of $X$ has a vertex in $Y$.

Proof. Let $Y$ be the set of vertices in $V(G) \backslash A$ with at least ten neighbours in $A$. Let $A_{1}$ be the set of vertices in $A$ with at most two neighbours in $Y$, and let $A_{2}=A \backslash A_{1}$.

(1) $\left|A_{1}\right| \geq \frac{1}{2}|A|$.

For we may assume that $A_{2} \neq \emptyset$, and therefore $Y \neq \emptyset$. Consequently $|A \cup Y| \geq 11 \geq 3$. Let $H_{1}$ be the bipartite subgraph of $G$ with $V\left(H_{1}\right)=A \cup Y$ and edge set all edges of $G$ between $A$ and $Y$. Since $H_{1}$ is planar, simple, and bipartite, and has at least three vertices, it follows (from Euler's formula; this is elementary and well known) that $\left|E\left(H_{1}\right)\right| \leq 2\left|V\left(H_{1}\right)\right|-4$. But $\left|E\left(H_{1}\right)\right| \geq 10|Y|$, since every vertex in $Y$ has at least 10 neighbours in $A$, and so $10|Y| \leq 2(|A|+|Y|)-4$. Consequently $|Y| \leq \frac{1}{4}|A|$. Now let $H_{2}$ be the subgraph of $H_{1}$ induced on $A_{2} \cup Y$. Then $\left|V\left(H_{2}\right)\right| \geq 4$ (since $A_{2} \neq \emptyset$ ), and therefore $\left|E\left(H_{2}\right)\right| \leq 2\left(\left|A_{2}\right|+|Y|\right)-4$ as before; but $\left|E\left(H_{2}\right)\right| \geq 3\left|A_{2}\right|$ since every member of $A_{2}$ has at least three neighbours in $Y$, and so $3\left|A_{2}\right| \leq 2\left(\left|A_{2}\right|+|Y|\right)-4$, and in particular, $\left|A_{2}\right| \leq 2|Y|$. Since $|Y| \leq \frac{1}{4}|A|$ as we already saw, it follows that $\left|A_{2}\right| \leq \frac{1}{2}|A|$, and therefore $\left|A_{1}\right| \geq \frac{1}{2}|A|$. This proves (1).

Let $H_{3}$ be the graph with vertex set $A_{1}$, in which distinct $u, v$ are adjacent if there is a path in $G \backslash Y$ between $u, v$ of length two. (Since $A$ is stable, it follows that no internal vertex of such a path is in $A$.) Since each vertex of $A$ has degree at most $d$ in $G$, and each vertex of $V(G) \backslash(A \cup Y)$ has at most nine neighbours in $A$, it follows that for each $v \in A$ there are at most $8 d$ paths in $G \backslash Y$ of length two with one end $v$, and hence $v$ has degree at most $8 d$ in $H_{3}$. Consequently $H_{3}$ is colourable with $8 d+1$ colours; and therefore there is a subset $A_{3}$ of $A_{1}$ such that $A_{3}$ is stable in $H_{3}$ and $\left|A_{3}\right| \geq\left|A_{1}\right| /(8 d+1)$. But by (1), $\left|A_{1}\right| \geq \frac{1}{2}|A|$, and so $\left|A_{3}\right| \geq|A| /(16 d+2)$. Let $H_{4}$ be the graph with vertex set $A_{3}$, in which distinct $u, v \in A_{3}$ are adjacent if there is a path in $G$ between $u, v$ of length 3 with no vertex in $Y$. Since no two members of $A_{3}$ have a common neighbour in $G \backslash Y$, this graph is a subgraph of the graph obtained from $G \backslash Y$ by contracting every edge with an end in $A_{3}$. In particular, $H_{4}$ is planar, and since it is simple, it is 4-colourable, and so there is a subset $A_{4} \subseteq A_{3}$ with $\left|A_{4}\right| \geq\left|A_{3}\right| / 4$ that is stable in $H_{4}$. Consequently, in $G$ every path between two members of $A_{4}$ has a vertex in $Y$. But

$$
\left|A_{4}\right| \geq \frac{1}{4}\left|A_{3}\right| \geq|A| /(64 d+8),
$$

and so setting $X=A_{4}$ satisfies the theorem. This proves 3.2. 
Now we prove the main result of this section.

Proof of 3.1. Let $G$ be a C4C planar cubic graph with $n$ vertices. Take a drawing of $G$ in a 2 -sphere $\Sigma$. Let us say a domino of $G$ is a closed disc $\Delta \subseteq \Sigma$ with boundary a cycle of $G$ of even length, such that $\Delta$ includes at most two regions of $G$, either exactly one region of $G$ of even length, or two regions of odd length.

(1) There exist at least $n / 32$ dominos of $G$, pairwise disjoint, and each with boundary of length at most 15 .

For let $G$ have $R$ regions; thus by Euler's formula, $R=n / 2+2$. Since the dual graph $G^{*}$ of $G$ is a 4-connected planar triangulation, Whitney's theorem [5] implies that the dual graph is Hamiltonian, and therefore we can number the regions of $G$ as $r_{1}, \ldots, r_{R}$, where for $1 \leq i \leq R$, the boundaries of $r_{i}, r_{i+1}$ share an edge (reading subscripts modulo $R$ ). We choose the numbering of the regions such that $r_{R}$ is the region of greatest length (the "length" of a region is the number of edges incident with it). Let $k=\left\lfloor\frac{1}{2} R\right\rfloor$. Then the average length of $r_{1}, \ldots, r_{2 k}$ is at most that of $r_{1}, \ldots, r_{R}$, and consequently less than six. Now for $1 \leq i \leq k$, the closure of one of $r_{2 i-1}, r_{2 i}, r_{2 i-1} \cup r_{2 i}$ is a domino $\Delta_{i}$ say, and its length is at most the sum of the lengths of $r_{2 i-1}$ and $r_{2 i}$ minus two. Thus the average length of $\Delta_{1}, \ldots, \Delta_{k}$ is less than 10. We claim that at least half of them have length at most 15 . For let $a$ of them have length at most 15, and $b$ length at least 16, and let $L$ be the sum of all their lengths. Then $10(a+b)>L$; but since they all have length at least four, $L \geq 4 a+16 b$. Consequently, $10(a+b)>4 a+16 b$, and so $a>b$. Thus at least half of $\Delta_{1}, \ldots, \Delta_{k}$ have length at most 15 . If we say that two of these dominos are adjacent when their boundaries share an edge, this defines a loopless planar graph, which is therefore 4-colourable; and consequently we can choose a quarter of our $\frac{1}{2} k$ dominos pairwise disjoint. Since $k \geq \frac{1}{2}(R-1) \geq \frac{1}{4} n$, this proves (1).

Let $A$ be a set of disjoint dominos as in (1), with $|A| \geq n / 32$. Let $R$ be the set of all regions of $G$ not included in any member of $A$. Let $H$ be the graph with $V(H)=A \cup R$, in which $\Delta \in A$ and $r \in R$ are adjacent if the boundaries of $\Delta$ and $r$ share an edge, and distinct $r_{1}, r_{2} \in R$ are adjacent if their boundaries share an edge. Thus $H$ is simple, and planar. By 3.2 with $d=15$, there exist $X \subseteq A$ and $Y \subseteq R$, such that

- $|X| \geq|A| / 968 \geq n / 30976$

- each member of $X$ is adjacent to at most two members of $Y$

- every path in $H$ of length at most three between two members of $X$ has a vertex in $Y$.

Let $X=\left\{\Delta_{1}, \ldots, \Delta_{k}\right\}$ say, where $k \geq n / 30976$. For $1 \leq i \leq k$, let $C_{i}$ be the cycle that forms the boundary of $\Delta_{i}$. There are two bracelets on $C_{i}$, and we choose one, $w_{i}$ say, as follows. There are at most two members of $Y$ adjacent to $\Delta_{i}$ in $H$. If there is at most one, let $w_{i}$ be an arbitrary bracelet on $C_{i}$. Suppose there are exactly two, say $r_{1}, r_{2}$. Thus the boundaries of $r_{1}, r_{2}$ both share at least one edge with the boundary of $\Delta_{i}$. If possible, choose a bracelet $w_{i}$ on $C_{i}$ such that for some edge $e$ of $G$, and for some $j \in\{1,2\}, w_{i}(e)=1$, and $e$ is the unique edge in common between the boundaries of $\Delta_{i}$ and $r_{j}$. If this is not possible, and so both $r_{1}, r_{2}$ are incident with two edges that belong to the boundary of $\Delta_{i}$, let $w_{i}$ be an arbitrary bracelet on $C_{i}$. 
We claim that the set $\left\{w_{1}, \ldots, w_{k}\right\}$ is a jewel-box. Let $W \subseteq\{1, \ldots, k\}$, and let $w$ be the sum of the looks of all $w_{i}(i \in W)$; we need to show that $w$ is a good look. It is certainly a look; let us check that it is good, that is, $w(D) \neq 1-|D|$ for every cut $D$ of $G$. Suppose not; and choose a cut $D$ of $G$ with $|D|$ minimal such that $w(D)=1-|D|$. Consequently $w(f)=0$ for some edge $f \in D$, and $w(e)=-1$ for all other edges of $D$. If some proper subset $D_{1}$ of $D$ is also a cut, then so is $D \backslash D_{1}$, and so we may assume that $f \in D_{1}$; but then $w\left(D_{1}\right)=1-\left|D_{1}\right|$ contrary to the minimality of $D$. Thus no proper subset of $D$ is a cut, and so $D$ is a bond of $G$ (that is, a minimal nonempty cut) and in particular, there is a cycle $C$ of the dual graph $G^{*}$ of $G$ with $E(C)=D$ (we identify $E\left(G^{*}\right)$ with $E(G)$ in the natural way). We recall that $R$ is the set of regions of $G$ not included in any member of $A$. Let $S$ be the set of all other regions of $G$; thus, $R, S$ form a partition of $V\left(G^{*}\right)$. For $i=-1,0,1$, let $F_{i}$ be the set of edges $e$ of $G$ with $w(e)=i$; thus $F_{-1}, F_{0}, F_{1}$ form a partition of $E(G)=E\left(G^{*}\right)$. We have seen that every edge of $C$ belongs to $F_{-1}$ except for one in $F_{0}$.

(2) If $s \in V(C) \cap S$, and $r_{1}, r_{2}$ are its neighbours in $C$, then not both $r_{1}, r_{2} \in Y$.

For let $e_{i}$ be the edge $r_{i} s$ of $G^{*}$. Suppose that $r_{1}, r_{2} \in Y$ and hence $r_{1}, r_{2} \in R$. Since $s \in S$, there is a unique $\Delta \in A$ such that $s$ is included in $\Delta$. Since $e_{1}, e_{2} \in E(C)$, at least one of them belongs to $F_{-1}$; and so $\Delta \in X$, say $\Delta=\Delta_{j}$ where $1 \leq j \leq k$, and $j \in W$. Consequently $e_{1}, e_{2}$ both belong to the supporting cycle of $w_{j}$, and so $w_{j}\left(e_{1}\right)=w_{j}\left(e_{2}\right)=-1$. From the choice of $w_{j}$ it follows that $r_{1}, r_{2}$ are both incident with two edges of the boundary of $\Delta_{j}$ in $G$; and since $G$ is 3-connected, it follows that $\Delta_{j}$ is not the closure of a region of $G$, and so $\Delta_{j}$ is the closure of the union of $s$ and some other region $s^{\prime}$ of $G$, and their boundaries share an edge $v_{1} v_{2}$ of $G$ that is drawn in the interior of $\Delta_{j}$ in the drawing of $G$. In particular, $s$ has odd length. Moreover, for $i=1,2$, the boundary of $r_{i}$ meets that of $s$ and that of $s^{\prime}$; and since $G$ is $\mathrm{C} 4 \mathrm{C}$ it follows that $r_{i}$ is incident with exactly one of $v_{1}, v_{2}$ in the drawing of $G$. Thus we may assume that $r_{1}$ is incident with $v_{1}$ and $r_{2}$ with $v_{2}$. Consequently for $i=1,2, e_{i}$ is incident with $v_{i}$ in $G$; and yet $w_{j}\left(e_{1}\right)=w_{j}\left(e_{2}\right)=-1$, contradicting that $s$ has odd length. This proves (2).

(3) Let $e$ be the unique edge of $C$ in $F_{0}$. Then in $G^{*}$, either both ends of e are in $R$ or both are in $S$.

For suppose that $e=r s$ in $G^{*}$, where $r \in R$ and $s \in S$. Let $r^{\prime}$ be the second neighbour of $s$ in $C$. Since $r^{\prime} s \in F_{-1}$, it follows that $r^{\prime} \in R$, and $s$ is included in some $\Delta_{i}$ where $i \in W$. But then every edge of the boundary of $\Delta_{i}$ belongs to $F_{1} \cup F_{-1}$, contradicting that $e \in F_{0}$. This proves (3).

(4) Let $s, s^{\prime} \in S$ both be incident with some edge e of $G$, so e $=s^{\prime}$ in $G^{*}$. If both $s, s^{\prime} \in V(C)$ then they are adjacent in $C$.

Let $P_{1}, P_{2}$ be the two paths of $C$ between $s, s^{\prime}$. In one of them, say $P_{1}$, every edge belongs to $F_{-1}$. Since in the drawing of $G$, the edge $e$ is drawn in the interior of a member of $A$, it follows that $e \in F_{0}$; and so the cycle $C_{1}$ of $G^{*}$ made by adding $e$ to $P_{1}$ satisfies $w\left(E\left(C_{1}\right)\right)=1-\left|E\left(C_{1}\right)\right|$. By the minimality of $|D|$, it follows that the length of $C_{1}$ is at least that of $C$, and so $P_{2}$ has only one edge, and hence $s, s^{\prime}$ are adjacent in $C$. This proves (4). 
Now every edge of $F_{-1}$ has (in $G^{*}$ ) an end in $R$ and an end in $S$, and consequently every edge of $C$ except one is between $R$ and $S$. Since $G$ is 3 -connected, it follows that $G^{*}$ is simple, and so $C$ has length at least three. By (3), $C$ has odd length, say $2 t+1$. Suppose that two consecutive vertices of $C$ belong to $S$; then we can number the vertices of $C$ in order as

$$
s_{0}-r_{1}-s_{1}-r_{2}-s_{2}-\cdots-r_{t}-s_{t^{-}} s_{0}
$$

where $r_{1}, \ldots, r_{t} \in R$ and $s_{0}, s_{1}, \ldots, s_{t} \in S$. For $1 \leq i \leq t$ there exists $\Delta \in A$ with $s_{i} \subseteq \Delta$, and since the edge $r_{i} s_{i}$ of $G^{*}$ belongs to $F_{-1}$, it follows that $\Delta=\Delta_{j}$ for some $j \in W$. Moreover, by (4) all these dominos are distinct, and so we may assume that $s_{i} \subseteq \Delta_{i}$ and $i \in W$ for $1 \leq i \leq t$. Suppose that $t \geq 2$. Then for $i=1,2$, the vertex $r_{i}$ of $H$ is adjacent in $H$ to the vertices $\Delta_{i}, \Delta_{i+1}$ of $H$, and so $r_{i} \in Y$ from the choice of $X, Y$; but this contradicts (2). It follows that $t=1$, and so $r_{1}$ is incident with an edge of the boundary of $s_{0}$, and an edge of the boundary of $s_{1}$. Since $G$ is C4C, it follows that these two edges have a common end in $G$, and therefore do not both belong to $F_{-1}$, a contradiction. This proves that no two consecutive vertices of $C$ belong to $S$.

Consequently there are two consecutive vertices of $C$ that belong to $R$, and we can number the vertices of $C$ in order as

$$
r_{0}-s_{1}-r_{1}-s_{2}-\cdots-s_{t}-r_{t}-r_{0}
$$

where $r_{0}, r_{1}, \ldots, r_{t} \in R$ and $s_{1}, \ldots, s_{t} \in S$. As before we may assume that $s_{i} \subseteq \Delta_{i}$ and $i \in W$ for $1 \leq i \leq t$. Suppose that $t \geq 3$; then for $i=1,2$, the vertex $r_{i}$ of $H$ is adjacent in $H$ to the vertices $\Delta_{i}, \Delta_{i+1}$ of $H$, and so $r_{i} \in Y$ from the choice of $X, Y$; but this contradicts (2). So $t \leq 2$. Suppose that $t=2$; then since there is a path of $H$ with vertices $\Delta_{1}, r_{2}, \Delta_{2}$, it follows that $r_{2} \in Y$ from the choice of $X, Y$; and since also there is a path of $H$ with vertices $\Delta_{2}, r_{0}, r_{1}, \Delta_{1}$ in order, it follows from the choice of $X, Y$ that at least one of $r_{0}, r_{1} \in Y$, contrary to (2). Thus $t=1$. But then $r_{0}, r_{1}, s_{1}$ are regions that pairwise are incident with a common edge, and since $G$ is $\mathrm{C} 4 \mathrm{C}$, it follows that for some vertex $v$ of $G$, these three edges are all incident with $v$, and yet two of the edges belong to $F_{-1}$, a contradiction. This proves that $\left\{w_{1}, \ldots, w_{k}\right\}$ is a jewel-box, and therefore completes the proof of 3.1 .

\section{The 3-connected case.}

In this section we extend 3.1 (changing the constant 30976 to something larger) to planar cubic graphs that are 3 -connected but not necessarily $\mathrm{C} 4 \mathrm{C}$. It would seem natural to do this by induction on the size of the graph, but we were not able to do so. Instead, we need to work with a set of three-edge cuts that decompose the graph into $\mathrm{C} 4 \mathrm{C}$ pieces, and we begin by describing this decomposition.

Let $G$ be a graph. A cut-decomposition of $G$ is a pair $(T, \phi)$ such that:

- $T$ is a tree with $E(T) \neq \emptyset$,

- $\phi: V(G) \rightarrow V(T)$ is a map, and

- for each $t \in V(T)$ with degree one or two in $T$, there exists $v \in V(G)$ with $\phi(v)=t$.

If $t \in V(T), \phi^{-1}(t)$ denotes the set of $v \in V(G)$ with $\phi(v)=t$. Similarly, if $Y \subseteq V(T)$, we denote $\{v \in V(G): \phi(v) \in Y\}$ by $\phi^{-1}(Y)$; and if $S$ is a subgraph of $T$ we write $\phi^{-1}(S)$ for $\phi^{-1}(V(S))$. For 
each edge $e$ of $T$, let $T_{1}, T_{2}$ be the two components of $T \backslash e$, and for $i=1,2$ let $X_{i}=\phi^{-1}\left(T_{i}\right)$. Thus $X_{1}, X_{2}$ is a partition of $V(G)$, and therefore $\delta\left(X_{1}\right)=\delta\left(X_{2}\right)$ is a cut; we denote this cut by $\phi^{-1}(e)$. If $\left|\phi^{-1}(e)\right|=k$ for each $e \in E(T)$ we call $(T, \phi)$ a $k$-cut-decomposition of $G$. We will only be concerned with 2- and 3-cut-decompositions of cubic graphs.

Let $(T, \phi)$ be a 3 -cut-decomposition of $G$, and let $T_{0}$ be a subtree of $T$. Let $T_{1}, \ldots, T_{s}$ be the components of $T \backslash V\left(T_{0}\right)$, and for $1 \leq i \leq s$ let $e_{i}$ be the unique edge of $T$ with an end in $V\left(T_{0}\right)$ and an end in $V\left(T_{i}\right)$. For $0 \leq i \leq s$, let $X_{i}=\phi^{-1}\left(T_{i}\right)$. Thus $X_{0}, X_{1}, \ldots, X_{s}$ are pairwise disjoint subsets of $V(G)$ with union $V(G)$. Let $G^{\prime}$ be the graph obtained from $G$ by, for $1 \leq i \leq s$, deleting all edges of $G \mid X_{i}$ and identifying all the vertices in $X_{i}$. (If $G \mid X_{i}$ is connected, this is the same as contracting all edges of $G \mid X_{i}$.) Thus the graph $G^{\prime}$ has $\left|X_{0}\right|+s$ vertices, and all the vertices of $G^{\prime}$ not in $X_{0}$ have degree 3. We call $G^{\prime}$ the 3 - $h u b$ of $G$ at $T_{0}$ (with respect to $(T, \phi)$ ). If $t_{0} \in V(T)$, by the "3-hub of $G$ at $t_{0}$ " we mean the 3 -hub of $G$ at $T_{0}$, where $T_{0}$ is the subtree of $T$ with vertex set $\left\{t_{0}\right\}$. If $\mathcal{C}$ is a class of graphs and $(T, \phi)$ is a 3 -cut-decomposition of $G$, and for each $t \in V(T)$ the 3-hub of $G$ at $t$ belongs to $\mathcal{C}$, we say that $(T, \phi)$ is a 3 -cut-decomposition of $G$ over $\mathcal{C}$.

Let $\mathcal{C}_{4}$ be the class of $\mathrm{C} 4 \mathrm{C}$ planar cubic graphs. We begin with:

4.1 Every 3-connected planar cubic graph $G$ that is not $C 4 C$ admits a 3 -cut-decomposition over $\mathcal{C}_{4}$.

Proof. We proceed by induction on $|V(G)|$. Since $G$ is not $\mathrm{C} 4 \mathrm{C}$, there is a partition $X_{1}, X_{2}$ of $V(G)$ such that $\left|\delta\left(X_{1}\right)\right|=3$ and $\left|X_{1}\right|,\left|X_{2}\right|>1$. Since $G$ is 3-connected, it follows that $G\left|X_{1}, G\right| X_{2}$ are connected. Let $G_{1}$ be obtained from $G$ by contracting the edges of $G \mid X_{2}$, and let $x_{1}$ be the vertex of $G_{1}$ formed by identifying the vertices of $X_{2}$. Define $G_{2}, x_{2}$ similarly. Then $G_{1}, G_{2}$ are 3-connected, planar, cubic, and have fewer vertices than $G$. For $i=1,2$, if $G_{i}$ is $\mathrm{C} 4 \mathrm{C}$ let $T_{i}$ be a tree with one vertex $t_{i}$ and define $\phi_{i}(v)=t_{i}$ for each $v \in V\left(G_{i}\right)$; if $G_{i}$ is not C4C, let $\left(T_{i}, \phi_{i}\right)$ be a 3-cut-decomposition of $G_{i}$ over $\mathcal{C}_{4}$ (this exists from the inductive hypothesis), and let $t_{i}=\phi_{i}\left(x_{i}\right)$. Note that for $i=1,2$, if $t_{i}$ has degree zero in $T_{i}$, then $\left|\phi_{i}^{-1}\left(t_{i}\right)\right| \geq 4$ since $\phi_{i}^{-1}\left(t_{i}\right)=V\left(G_{i}\right)$ and $G_{i}$ is 3-connected; while if $t_{i}$ has degree one in $T_{i}$ then $\left|\phi_{i}^{-1}\left(t_{i}\right)\right| \geq 3$ since the 3-hub of $G_{i}$ at $t_{i}$ with respect to $\left(T_{i}, \phi_{i}\right)$ is $\mathrm{C} 4 \mathrm{C}$. Thus in either case $\left|\phi_{i}^{-1}\left(t_{i}\right)\right| \geq 3$. Let $T$ be the tree obtained from the disjoint union of $T_{1}$ and $T_{2}$ by making $t_{1}, t_{2}$ adjacent; and for $v \in V(G)$, define $\phi(v)=\phi_{i}(v)$ where $v \in X_{i}$. Consequently, for $i=1,2$, if $t_{i}$ has degree at most two in $T$ then $\left|\phi^{-1}\left(t_{i}\right)\right| \geq 2$. It is easy to check that $(T, \phi)$ is a 3 -cut-decomposition of $G$ over $\mathcal{C}_{4}$. This proves 4.1.

4.2 Let $(T, \phi)$ be a 3-cut-decomposition of a 3-connected cubic graph $G$, let $T_{1}, \ldots, T_{k}$ be pairwise vertex-disjoint subtrees of $T$, and for $1 \leq i \leq k$ let $H_{i}$ be the 3 -hub of $G$ at $T_{i}$. For $1 \leq i \leq k$ let $\mathcal{B}_{i}$ be a set of bracelets of $G$, such that

- $\mathcal{B}_{i}$ is a jewel-box of $H_{i}$, and

- if $C$ is the supporting cycle of a member of $\mathcal{B}_{i}$, then $V(C) \subseteq \phi^{-1}\left(T_{i}\right)$.

Then $\mathcal{B}_{1} \cup \cdots \cup \mathcal{B}_{k}$ is a jewel-box in $G$.

Proof. Let $w, w^{\prime} \in \mathcal{B}_{1} \cup \cdots \cup \mathcal{B}_{k}$ be distinct, on cycles $C, C^{\prime}$ of $G$ respectively. We must show that $C, C^{\prime}$ are vertex-disjoint. Choose $i, j \in\{1, \ldots, k\}$ such that $w \in \mathcal{B}_{i}$ and $w^{\prime} \in \mathcal{B}_{j}$. Thus $V(C) \subseteq \phi^{-1}\left(T_{i}\right)$ and $V\left(C^{\prime}\right) \subseteq \phi^{-1}\left(T_{j}\right)$. If $i \neq j$ then $T_{i}, T_{j}$ are disjoint and therefore $C, C^{\prime}$ are disjoint; while if $i=j$ then $w, w^{\prime}$ both belong to the same jewel-box in $H_{i}$, and therefore have disjoint supporting cycles. 
Second, let $W \subseteq \mathcal{B}_{1} \cup \cdots \cup \mathcal{B}_{k}$, and let $w$ be the sum of the looks of all members of $W$; and for $i=-1,0,1$ let $F_{i}$ be the set of edges $e$ of $G$ such that $w(e)=i$. We must show that $w$ is a good look. Suppose not; then there is a cut $D$ of $G$ and an edge $f \in D$ such that $f \in F_{0}$ and $D \backslash\{f\} \subseteq F_{-1}$. Choose such a cut $D$ with $|D|$ minimum. Then as in the proof of $3.1, D$ is a bond of $G$, that is, $D=\delta(X)$ for some $X \subseteq V(G)$ with $G|X, G|(V(G) \backslash X)$ both connected.

(1) Let $e \in E(T)$, let $S_{1}, S_{2}$ be the two components of $T \backslash e$, and for $i=1,2$ let $Y_{i}=\phi^{-1}\left(V\left(S_{i}\right)\right)$. Then one of $X \cap Y_{1}, X \cap Y_{2}, Y_{1} \backslash X, Y_{2} \backslash X$ is empty.

Suppose all four of these sets are nonempty. Since $\delta\left(Y_{1}\right) \subseteq F_{0}$, and $\delta(X) \backslash\{f\} \subseteq F_{-1}$, it follows that $\delta\left(Y_{1}\right) \cap \delta(X) \subseteq\{f\}$. There are two cases depending whether $f \in \delta\left(Y_{1}\right)$ or not. Suppose first that $f \notin \delta\left(Y_{1}\right)$. Then $\delta\left(Y_{1}\right) \cap \delta(X)=\emptyset$, and from the symmetry between $Y_{1}$ and $Y_{2}$ we may assume that both ends of $f$ belong to $Y_{2}$. Since $G \mid X$ is connected and $X \cap Y_{1}, X \cap Y_{2}$ are both nonempty, some edge of $\delta\left(Y_{1}\right)$ has both ends in $X$, and similarly some edge of $\delta\left(Y_{1}\right)$ has both in $V(G) \backslash X$. Since $\left|\delta\left(Y_{1}\right)\right|=3$ and every edge of $\delta\left(Y_{1}\right)$ either has both ends in $X$ or both in $V(G) \backslash X$, we may assume (replacing $X$ by its complement if necessary) that exactly one edge $f^{\prime}$ of $\delta\left(Y_{1}\right)$ has both ends in $X$. But then $f^{\prime} \in F_{0}$, and $f^{\prime}$ belongs to the cut $\delta\left(X \cap Y_{1}\right)$, and every other edge of this cut belongs to $\delta(X)$ and therefore to $F_{-1}$. From the minimality of $|D|$, it follows that $\left|\delta\left(X \cap Y_{1}\right)\right| \geq|\delta(X)|$, and so there is at most one edge of $G$ between $X \cap Y_{2}$ and $Y_{2} \backslash X$. But then $\left|\delta\left(X \cap Y_{2}\right)\right| \leq 2$, a contradiction since $G$ is 3 -connected.

This proves that $f \in \delta\left(Y_{1}\right)$. From the symmetry between $Y_{1}, Y_{2}$ we may assume that $f$ is between $X \cap Y_{2}$ and $Y_{1} \backslash X$. Since $G \mid X$ is connected, there is at least one edge between $X \cap Y_{1}$ and $X \cap Y_{2}$. Suppose that there is only one. Since $G$ is 3-connected, there are at least two edges between $X \cap Y_{1}$ and $Y_{1} \backslash X$, and an edge between $X \cap Y_{2}$ and $Y_{2} \backslash X$; and so $\delta\left(X \cap Y_{1}\right)$ contradicts the minimality of $|D|$. Thus there are at least two edges between $X \cap Y_{1}$ and $X \cap Y_{2}$. Since $\left|\delta\left(Y_{1}\right)\right|=3$, there are no edges between $Y_{1} \backslash X$ and $Y_{2} \backslash X$, contradicting that $G \mid(V(G) \backslash X)$ is connected. This proves (1).

Since $G$ is 3 -connected and therefore $|D| \geq 3$, we may choose $e \in D$ with $e \neq f$. Thus $\phi(e)=-1$, and so $e$ is an edge of one of $C_{1}, \ldots, C_{k}$, say $C_{1}$. Let $Y_{0}=\phi^{-1}\left(T_{1}\right)$. Let $e=x y$; then since $V\left(C_{1}\right) \subseteq Y_{0}$ it follows that $x, y \in Y_{0}$, and since $e \in \delta(X)$, we deduce that $X \cap Y_{0}, Y_{0} \backslash X$ are both nonempty. Let $S_{1}, \ldots, S_{s}$ be the components of $T \backslash V\left(T_{1}\right)$, and for $1 \leq i \leq s$ let $e_{i}$ be the unique edge of $T$ with an end in $V\left(T_{1}\right)$ and an end in $V\left(S_{i}\right)$. For $1 \leq i \leq s$, let $Y_{i}=\phi^{-1}\left(S_{i}\right)$. Thus $Y_{0}, Y_{1}, \ldots, Y_{s}$ are pairwise disjoint subsets of $V(G)$ with union $V(G)$. The 3-hub $H_{1}$ of $G$ at $T_{1}$ is therefore obtained by contracting all edges of $G \mid Y_{i}$ for $1 \leq i \leq s$. Since $D$ is not a cut of $H_{1}$ (because $\mathcal{B}_{1}$ is a jewel-box of $H_{1}$ ) it follows that for some $i \in\{1, \ldots, s\}$, both $X \cap Y_{i}, Y_{i} \backslash X$ are nonempty. But since both $Y_{0} \cap X, Y_{0} \backslash X$ are nonempty, this contradicts (1) applied to the edge $e_{i}$. This proves 4.2.

Here is a convenient lemma:

4.3 Let $G$ be a cubic graph, and let $F_{1}, F_{2}, F_{3}$ be pairwise disjoint perfect matchings of $G$. Let $C_{1}, \ldots, C_{k}$ be the cycles of $G$ with edge-set included in $F_{1} \cup F_{2}$, and for $1 \leq i \leq k$ let $w_{i}$ be the bracelet on $C_{i}$ such that $w_{i}(e)=-1$ if $e \in F_{1} \cap E\left(C_{i}\right)$ and $w_{i}(e)=1$ if $e \in F_{2} \cap E\left(C_{i}\right)$. Then $\left\{w_{1}, \ldots, w_{k}\right\}$ is a jewel-box in $G$, and so $\beta(G) \geq k$. Moreover, if $e \in E(G)$, then $\beta(G)>0$, and there is a bracelet $w$ such that $e$ is not in the supporting cycle of $w$ and $\{w\}$ is a jewel-box.

Proof. We must show that for every subset $X \subseteq F_{1} \cup F_{2}$ that is a union of edge-sets of cycles, if we delete from $G$ every edge in $F_{1} \cap X$, and add a new edge parallel to every edge in $F_{2} \cap X$, then 
the graph $G^{\prime}$ that we construct has no cutedge. But $F_{2}, F_{3}$ are disjoint perfect matchings of $G^{\prime}$, and the remaining edges of $G^{\prime}$ form a third, and so $G^{\prime}$ is 3-edge-colourable and therefore has no cutedge. Consequently $\left\{w_{1}, \ldots, w_{k}\right\}$ is a jewel-box, and so $\beta(G) \geq k$. For the final claim, let $e \in E(G)$; from the symmetry between $F_{1}, F_{2}, F_{3}$ we may assume that $e \in F_{3}$, and since $F_{1}, F_{2} \neq \emptyset$ there is a cycle within $F_{1} \cup F_{2}$, so the final claim follows from the first. This proves 4.3.

A 3-edge-colouring of $G$ is a map $\kappa: E(G) \rightarrow\{1,2,3\}$ such that $\kappa(e) \neq \kappa(f)$ for every two distinct edges $e, f \in E(G)$ with a common end; and two 3-edge-colourings $\kappa, \kappa^{\prime}$ are equivalent if there is a permutation $\pi$ of $\{1,2,3\}$ such that $\kappa^{\prime}(e)=\pi(\kappa(e))$ for each $e \in E(G)$. If $\kappa$ is a 3-edge-colouring of $G$ and $X \subseteq E(G)$, we denote the restriction of $\kappa$ to $X$ by $\kappa \mid X$; and if $i \in\{1,2,3\}$, we denote the set of $e \in E(G)$ with $\kappa(e)=i$ by $\kappa^{-1}(i)$. A cubic graph $G$ is uniquely 3 -edge-colourable (U3C) if there is a unique set $\left\{F_{1}, F_{2}, F_{3}\right\}$ of perfect matchings of $G$ with union $E(G)$; that is, if it is 3-edge-colourable and all its 3-edge-colourings are equivalent.

Finding a 3-edge-colouring of a cubic graph $G$ equipped with a 3 -cut-decomposition is just a matter of finding a 3-edge-colouring of the 3-hub of $G$ at each vertex of the tree. In particular, if $(T, \phi)$ is a 3 -cut-decomposition of a cubic graph $G$, and $H$ is the 3-hub of $G$ at some $t \in V(T)$, and $\kappa$ is a 3-edge-colouring of $G$, then $\kappa \mid E(H)$ is a 3-edge-colouring of $H$; while if $G$ admits a 3-edge-colouring, then every 3-edge-colouring of $H$ can be extended to a 3-edge-colouring of $G$.

If $(T, \phi)$ is a 3 -cut-decomposition of a cubic graph $G$ with $|V(T)| \geq 3$, and $e=t_{1} t_{2}$ is an edge of $T$, let $T^{\prime}$ be the tree obtained from $T$ by contracting $e$ (forming a vertex $t^{\prime}$ say), and for $v \in V(G)$, define $\phi^{\prime}(v)=\phi(v)$ if $\phi(v) \neq t_{1}, t_{2}$, and $\phi^{\prime}(v)=t^{\prime}$ if $\phi(v)=t_{1}$ or $t_{2}$. Then $\left(T^{\prime}, \phi^{\prime}\right)$ is also a 3-cutdecomposition of $G$, and we say it is obtained from $(T, \phi)$ by contracting e. Note that if the 3-hub of $G$ at one of $t_{1}, t_{2}$ (with respect to $(T, \phi)$ ) is not $\mathrm{U} 3 \mathrm{C}$, then the 3 -hub of $G$ at $t^{\prime}$ (with respect to $\left.\left(T^{\prime}, \phi^{\prime}\right)\right)$ is not $\mathrm{U} 3 \mathrm{C}$, since if the second 3-hub is 3-edge-colourable then every 3-edge-colouring of the first 3 -hub extends to a 3 -edge-colouring of the second.

4.4 Let $(T, \phi)$ be a 3-cut-decomposition of a 3-connected 3-edge-colourable cubic graph $G$. Then there are at most $6 \beta(G)$ vertices $t \in V(T)$ such that the 3 -hub of $G$ at $t$ is not U3C.

Proof. Let there be $n_{1}$ vertices $t \in V(T)$ such that the 3 -hub of $G$ at $t$ is not U3C; we need to show that $n_{1} \leq 6 \beta(G)$. Since $\beta(G) \geq 1$ by 4.3 , we may assume that $n_{1} \geq 7$. By contracting edges of $T$ appropriately, we may therefore assume that for every vertex $t \in V(T)$, the 3-hub of $G$ at $t$ is not U3C. Consequently we may choose 3-edge-colourings $\kappa, \kappa^{\prime}$ of $G$, such that for each $t \in V(T)$ with 3-hub $H$ say, $\kappa \mid E(H)$ and $\kappa^{\prime} \mid E(H)$ are not equivalent. By permuting the elements of $\{1,2,3\}$ in one of $\kappa, \kappa^{\prime}$, we may assume that $\kappa\left|\phi^{-1}(e)=\kappa^{\prime}\right| \phi^{-1}(e)$ for at least one-sixth of all edges $e \in E(T)$. By contracting all other edges of $T$, we obtain a 3-cut-decomposition $(S, \psi)$ of $G$, such that

- $|E(S)| \geq\left(n_{1}-1\right) / 6$,

- for each $t \in V(S)$ with 3-hub $H$ say, $\kappa\left|E(H) \neq \kappa^{\prime}\right| E(H)$,

- $\kappa\left|\psi^{-1}(e)=\kappa^{\prime}\right| \psi^{-1}(e)$ for all $e \in E(S)$.

Let $V(S)=\left\{s_{1}, \ldots, s_{m}\right\}$ say, where $m \geq\left(n_{1}-1\right) / 6+1$, and for $1 \leq i \leq m$ let $H_{i}$ be the 3-hub of $G$ at $s_{i}$ with respect to $(S, \psi)$. Fix $i$ with $1 \leq i \leq m$. For $j=1,2,3$, let $F_{j}$ be the set of edges $e$ of $H_{i}$ with $\kappa(e)=j$, and let $F_{j}^{\prime}$ be the set of edges $e$ of $H$ with $\kappa^{\prime}(e)=j$. Since $\kappa\left|E\left(H_{i}\right) \neq \kappa^{\prime}\right| E\left(H_{i}\right)$, there exists $j \in\{1,2,3\}$ such that $F_{j} \neq F_{j}^{\prime}$. Choose a cycle $C_{i}$ with $E\left(C_{i}\right) \subseteq\left(F_{j} \backslash F_{j}^{\prime}\right) \cup\left(F_{j}^{\prime} \backslash F_{j}\right)$, 
and let $w_{i}$ be some bracelet on $C_{i}$. Note that $\psi(v)=s_{i}$ for every $v \in V\left(C_{i}\right)$, since every edge of $C$ belongs to just one of $F_{j}, F_{j}^{\prime}$, and yet $\kappa\left|\psi^{-1}(e)=\kappa^{\prime}\right| \psi^{-1}(e)$ for every edge $e$ of $S$. We claim that the look of $w_{i}$ is a good look in $H_{i}$. To see this, let $J$ be obtained from $H_{i}$ by deleting every edge $e$ with $w_{i}(e)=-1$, and adding a new edge parallel to every edge $e$ with $w(e)=1$; we must show that $J$ has no cutedge. Since every edge of $C_{i}$ belongs to exactly one of $F_{j}, F_{j}^{\prime}$, we may assume that the edges $e$ of $C_{i}$ with $w_{i}(e)=-1$ belong to $F_{j}$, and that $j=1$ say. But then $F_{2}, F_{3}$ are disjoint perfect matchings of $J$, and the remaining edges of $J$ form a third perfect matching, and so $J$ is 3 -edge-colourable, and therefore has no cutedge. This proves that the look of $w_{i}$ is a good look in $H_{i}$, and so $\left\{w_{i}\right\}$ is a jewel-box in $H_{i}$.

These jewel-boxes satisfy the hypotheses of 4.2 , and so by 4.2 , it follows that the set $\left\{w_{1}, \ldots, w_{m}\right\}$ is a jewel-box in $G$. Since

$$
\beta(G) \geq m \geq\left(n_{1}-1\right) / 6+1 \geq n_{1} / 6
$$

this proves 4.4 .

We need the following theorem of Fowler [1]:

\subsection{The only planar cubic graph that is both $C_{4} C$ and $U 3 C$ is the graph $K_{4}$.}

Let $u_{1}, u_{2}, u_{3}$ be pairwise adjacent vertices of a cubic graph $G^{\prime}$, and for $1 \leq i \leq 3$ let $u_{i}$ have a neighbour $v_{i} \notin\left\{u_{1}, u_{2}, u_{3}\right\}$. Let $G$ be obtained from $G^{\prime}$ by contracting the three edges $u_{1} u_{2}, u_{2} u_{3}, u_{3} u_{1}$, forming a vertex $v$ say. We say that $G^{\prime}$ is obtained from $G$ by "replacing $v$ by a triangle". Let $w$ be a bracelet in $G$, with supporting cycle $C$. If $v \notin V(C)$ then $w$ is a bracelet in $G^{\prime}$. If $v \in V(C)$, and say the edges $v v_{1}, v v_{2}$ belong to $E(C)$, let $C^{\prime}$ be the cycle of $G^{\prime}$ consisting of the path $C \backslash v$ and the path $v_{1}-u_{1}-u_{3}-u_{2}-v_{2}$, and let $w^{\prime}$ be the bracelet on $C^{\prime}$ that equals $w$ on the edges of $C \backslash v$. In this case we call $w^{\prime}$ the "natural rerouting of $w$ ".

4.6 Let $G, v, G^{\prime}, u_{1}, u_{2}, u_{3}, v_{1}, v_{2}, v_{3}$ be as above, and let $\mathcal{B}=\left\{w_{1}, \ldots, w_{k}\right\}$ be a jewel-box in $G$. For $1 \leq i \leq k$, if $v$ is in the supporting cycle of $w_{i}$ let $w_{i}^{\prime}$ be the natural rerouting of $w_{i}$, and otherwise let $w_{i}^{\prime}=w_{i}$. Let $\mathcal{B}^{\prime}=\left\{w_{1}^{\prime}, \ldots, w_{k}^{\prime}\right\}$. Then $\mathcal{B}^{\prime}$ is a jewel-box in $G^{\prime}$.

Proof. Certainly the members of $\mathcal{B}^{\prime}$ have disjoint supporting cycles, because $v$ belongs to the supporting cycle of at most one member of $\mathcal{B}$. Let $W^{\prime} \subseteq \mathcal{B}^{\prime}$, and let $w^{\prime}$ be the sum of the looks of members of $W^{\prime}$; we must show that $w^{\prime}$ is a good look in $G^{\prime}$. Suppose not, and let $D^{\prime}$ be a cut of $G^{\prime}$ with $w^{\prime}\left(D^{\prime}\right)=1-\left|D^{\prime}\right|$. Choose $X^{\prime} \subseteq V\left(G^{\prime}\right)$ with $D^{\prime}=\delta_{G^{\prime}}\left(X^{\prime}\right)$. By replacing $X^{\prime}$ by its complement if necessary, we may assume that at most one of $u_{1}, u_{2}, u_{3} \in X^{\prime}$. For $-1 \leq i \leq 1$, let $F_{i}$ be the set of edges $e \in E(G)$ with $w(e)=i$, and let $F_{i}^{\prime}$ be the set of edges $e \in E\left(G^{\prime}\right)$ with $w^{\prime}(e)=i$. Thus every edge of $D^{\prime}$ belongs to $F_{-1}^{\prime}$ except for exactly one in $F_{0}^{\prime}$. Let $W$ be the set of all $w_{i}$ such that $w_{i}^{\prime} \in W^{\prime}$, and let $w$ be the sum of the looks in $G$ of the members of $W$. Thus $w$ is a good look in $G$.

Suppose first that none of $u_{1}, u_{2}, u_{3} \in X^{\prime}$; then $D^{\prime}$ is also a cut of $G$, and $w\left(D^{\prime}\right)=w^{\prime}\left(D^{\prime}\right)=$ $1-\left|D^{\prime}\right|$, contradicting that $w$ is a good look in $G$. So we may assume that $u_{1} \in X^{\prime}$ and $u_{2}, u_{3} \notin X^{\prime}$. In particular, $u_{1} u_{2}, u_{1} u_{3} \in D^{\prime}$, and since $\left|D^{\prime} \backslash F_{-1}^{\prime}\right|=1$, we may assume that $u_{1} u_{2} \in F_{-1}^{\prime}$. Consequently there exists some member of $W^{\prime}$, say $w_{1}^{\prime}$, such that $u_{1} u_{2}$ is an edge of the supporting cycle of $w_{1}^{\prime}$, and $w_{1}^{\prime}\left(u_{1} u_{2}\right)=-1$; and so $w_{1} \in W$, and $v$ belongs to the supporting cycle of $w_{1}$. Now $u_{1} u_{3} \notin F_{-1}^{\prime}$ from the definition of $w^{\prime}$, and yet no edge of $D^{\prime}$ is in $F_{1}^{\prime}$; so $u_{1} u_{3} \in F_{0}^{\prime}$. It follows that $u_{2} u_{3} \in F_{1}^{\prime}$, since $u_{2} u_{3}$ is an edge of the supporting cycle of $w_{1}^{\prime}$; and so $u_{2} v_{2} \in F_{0} \cap F_{0}^{\prime}$, and $u_{3} v_{3} \in F_{-1} \cap F_{-1}^{\prime}$. Let $X=\left(X^{\prime} \backslash\left\{u_{1}\right\}\right) \cup\{v\}$, and let $D$ be the cut $\delta_{G}(X)$ of $G$; then every edge of $D$ belongs to $F_{-1}$ 
except for exactly one, namely $u_{2} v_{2} \in F_{0}$, contradicting that $w$ is a good look in $G$. This proves 4.6 .

A leaf of a tree means a vertex with degree one. The inner tree of a tree $T$ with at least three vertices is the tree obtained by deleting all leaves of $T$. An twig of a tree $T$ is a leaf of its inner tree. Thus, for every twig $t$ of $T, t$ has at least two neighbours in $T$, and all of its neighbours are leaves of $T$ except exactly one.

4.7 Let $G$ be a 3-connected 3-edge-colourable cubic graph, and let $(T, \phi)$ be a 3-cut-decomposition of $G$ over $\mathcal{C}_{4}$. Then $T$ has at most $7 \beta(G)$ twigs.

Proof. Let $P$ be the set of vertices $t$ of $T$ such that the 3 -hub at $t$ is not U3C.

(1) Let $t_{0}$ be a twig and $t_{1}, \ldots, t_{k}$ all the leaves adjacent to $t_{0}$. Let $S$ be the subtree of $T$ with vertex set $\left\{t_{0}, t_{1}, \ldots, t_{k}\right\}$, and suppose that $V(S) \cap P=\emptyset$. Let $H$ be the 3 -hub of $G$ at $S$. Then there is a cycle $C$ of $G$, satisfying $\phi(v) \in V(S)$ for every vertex $v \in V(C)$, and a bracelet $w$ on $C$, such that $\{w\}$ is a jewel-box of $H$.

For $0 \leq i \leq k$ let $H_{i}$ be the 3-hub of $G$ at $t_{i}$. Since $H_{i}$ is both $\mathrm{C} 4 \mathrm{C}$ and U3C, it follows from 4.5 that $H_{i}$ is isomorphic to $K_{4}$ for $0 \leq i, \ldots, k$. In particular, since $H_{0}$ is isomorphic to $K_{4}$, it follows that $1 \leq k \leq 3$. Let $H_{0}$ have vertex set $\left\{v_{1}, \ldots, v_{4}\right\}$, where $v_{4}$ is the vertex formed by identifying all vertices $v$ of $G$ with $\phi(v) \notin V(S)$, and for $1 \leq i \leq k v_{i}$ is the vertex formed by identifying the members of $\phi^{-1}\left(t_{i}\right)$. Thus $\left\{v_{k+1}, \ldots, v_{3}\right\}=\phi^{-1}\left(t_{0}\right)$. Consequently $H$ is obtained from $K_{4}$ by replacing $v_{1}, \ldots, v_{k}$ by triangles. Since $k \geq 1$, it follows that $H$ is obtained from a "prism" (the complement of a six-vertex cycle), say $J$, with one vertex called $v_{4}$, by replacing one or two other vertices by triangles. In view of 4.6, it suffices to check that there is a cycle $C$ of the prism $J$ with $v_{4} \notin V(C)$ such that $\{w\}$ is a jewel-box in $J$ for some bracelet $w$ on $C$. We leave this to the reader (use the cycle of length four). This proves (1).

Let there be $m$ twigs in $T$. The at least $m-|P|$ of them satisfy the hypotheses of (1), and so by 4.2, $\beta(G) \geq m-|P|$. But $|P| \leq 6 \beta(G)$ by 4.4 , and so $m \leq 7 \beta(G)$. This proves 4.7 .

4.8 Let $G$ be a 3-connected 3-edge-colourable cubic graph, and let $(T, \phi)$ be a 3-cut-decomposition of $G$ over $\mathcal{C}_{4}$. Then the inner tree of $T$ (if it exists) has at most $84 \beta(G)$ vertices.

Proof. Let $P$ be the set of vertices $t$ of $T$ such that the 3 -hub at $t$ is not U3C. Let $T^{\prime}$ be the inner tree of $T$ (we may assume that this exists). Let $t_{1}-\cdots-t_{4}$ be a four-vertex path of $T^{\prime}$ such that $t_{1}, \ldots, t_{4}$ all have degree two in $T^{\prime}$; and let $S$ be the subtree of $T$ induced on the union of $\left\{t_{1}, \ldots, t_{4}\right\}$ and the set of leaves of $T$ adjacent to one of $t_{1}, \ldots, t_{4}$. We call $S$ a limb of $T$.

(1) Let $S$ be a limb with $t_{1}, \ldots, t_{4}$ as above, and suppose that $P \cap V(S)=\emptyset$. Let $H$ be the 3hub of $G$ at $S$. Then there is a cycle $C$ of $G$ with $V(C) \subseteq \phi_{-1}(S)$, and a bracelet $w$ on $C$ such that $\{w\}$ is a jewel-box of $H$.

For since $P \cap V(S)=\emptyset$, it follows from 4.5 that the 3 -hub of $G$ at $t$ is isomorphic to $K_{4}$ for every vertex $t \in V(S)$; and so $t_{1}, \ldots, t_{4}$ each have degree two, three or four in $T$. It follows that the 
3-hub of $G$ at the subtree with vertex set $\left\{t_{1}, \ldots, t_{4}\right\}$ can be constructed as follows: start with $K_{4}$ and call one of its vertices $x$; choose another vertex of the $K_{4}$ and replace it by a triangle; choose a vertex of this triangle and replace it by a triangle; choose a vertex of the most recent triangle and replace it by a triangle; and choose a vertex of this latest triangle and call it $y$. Let us call this graph $J$. Then $H$ can be obtained from $J$ by replacing any of its vertices by triangles, except $x, y$. We must check that there is a cycle of $H$ not containing $x, y$, and a bracelet $w$ on $H$, such that $\{w\}$ is a jewel-box of $H$. By 4.6, it is enough to prove the claim for $J$ rather for $H$. To check the claim for $J$, note that any cycle of length four in $J$ not using $x, y$ will do, so we can assume there is no such cycle, and then the possibilities for $J$ are greatly restricted (there are only three). We leave the rest to the reader. This proves $(1)$.

Let $\left|V\left(T^{\prime}\right)\right|=m$ (and we may assume that $m>1$ ), and let $T^{\prime}$ have $m_{1}$ leaves, and $m_{2}$ vertices of degree two. Thus $T^{\prime}$ has $m-m_{1}-m_{2}$ vertices of degree at least three. Since every tree has at least as many leaves as it does vertices of degree at least three, it follows that $m-m_{1}-m_{2} \leq m_{1}$, and so $m \leq 2 m_{1}+m_{2}$. A branch of a tree is a maximal subpath (with at least one edge) such that all its internal vertices have degree two in the tree; the number of branches of a tree is one less than the number of vertices of degree different from two. Thus $T^{\prime}$ has $m-m_{2}-1$ branches. Let its branches be $B_{1}, \ldots, B_{k}$ say, where $k=m-m_{2}-1$, and let $B_{i}$ have $b_{i}+2$ vertices for $1 \leq i \leq k$. Thus $b_{1}+\cdots+b_{k}=m_{2}$. Each $B_{i}$ has $b_{i}$ internal vertices, and so there are $\left\lfloor b_{i} / 4\right\rfloor \geq\left(b_{i}-3\right) / 4$ disjoint 4 -vertex paths within the interior of $B_{i}$. Consequently there are at least

$$
\sum_{i=1, \ldots, k}\left(b_{i}-3\right) / 4 \geq m_{2} / 4-3 k / 4
$$

disjoint limbs in $T$, and at most $|P|$ of them contain members of $P$, so at least $m_{2} / 4-3 k / 4-|P|$ of them satisfy the hypotheses of (1). By 4.2 , it follows that $m_{2} / 4-3 k / 4-|P| \leq \beta(G)$. Since $k \leq m-m_{2}$, it follows that $4 m_{2}-3 m-4|P| \leq 4 \beta(G)$. We already saw that $m \leq 2 m_{1}+m_{2}$ (that is, $\left.m-8 m_{1} \leq 4 m_{2}-3 m\right)$, and therefore $m \leq 8 m_{1}+4|P|+4 \beta(G)$. But $m_{1} \leq 7 \beta(G)$ by 4.7 , and $|P| \leq 6 \beta(G)$ by 4.4 , and it follows that $m \leq 84 \beta(G)$. This proves 4.8 .

4.9 Let $H$ be a $C 4 C$ planar cubic graph, let $X \subseteq V(H)$, and let $G$ be obtained from $H$ by replacing some vertices not in $X$ by triangles. Then there is a jewel-box $\mathcal{B}$ in $G$ of cardinality at least $|V(G)| / 92928$ - $|X|$, such that the supporting cycle of each member of $\mathcal{B}$ contains no vertex in $X$.

Proof. By 3.1, there is a jewel-box in $H$ with cardinality at least $|V(H)| / 30976 \geq|V(G)| / 92928$. The supporting cycles of at most $|X|$ members of this jewel-box contain a vertex of $X$, and so there is a jewel-box in $H$ with cardinality at least $|V(G)| / 92928-|X|$ such that none of the supporting cycles of its members contain members of $X$. By 4.6, the same holds in $G$. This proves 4.9 .

\subsection{For every 3-connected planar cubic graph $G, \beta(G) \geq|V(G)| / 16819968$.}

Proof. If $G$ is $\mathrm{C} 4 \mathrm{C}$ this follows from 3.1, so we assume that $G$ is not C4C. By 4.1, there is a 3 -cut-decomposition $(T, \phi)$ of $G$ over $\mathcal{C}_{4}$. Let the vertices of $T$ be $t_{1}, \ldots, t_{k}$. Let $P$ be the set of vertices $t$ of $T$ such that the 3-hub of $G$ at $t$ is not U3C. Let $L$ be the set of all leaves of $T$ that do not belong to $P$, and let $S=T \backslash L$. If $S$ is null then $|L|=2$ and both members of $L$ have 
U3C 3-hubs, and so by $4.5|V(G)| \leq 6$ and the theorem holds. We may therefore assume that $S$ is nonnull. Let $V(S)=\left\{t_{1}, \ldots, t_{s}\right\}$ say, where $s \leq k$; and so $L=\left\{t_{s+1}, \ldots, t_{k}\right\}$. For $1 \leq i \leq s$, let $T_{i}$ be the subtree of $T$ with vertex set consisting of $t_{i}$ and all members of $L$ adjacent to $t_{i}$. Thus $T_{1}, \ldots, T_{s}$ are pairwise disjoint subtrees of $T$, and every vertex of $T$ belongs to exactly one of them. For $1 \leq i \leq s$, let $H_{i}$ be the 3-hub of $G$ at $T_{i}$. Then $V\left(H_{i}\right)$ is the disjoint union of two sets $X_{i}, Y_{i}$ say, where $Y_{i}=\phi^{-1}\left(T_{i}\right)$. Now $H_{i}$ is obtained from the 3 -hub of $G$ at $t_{i}$ (which is C4C) by replacing some vertices not in $X_{i}$ by triangles; and so by 4.9 there is a jewel-box $\mathcal{B}_{i}$ in $H_{i}$ of cardinality at least $\left|V\left(H_{i}\right)\right| / 92928-\left|X_{i}\right| \geq\left|Y_{i}\right| / 92928-\left|X_{i}\right|$, such that the supporting cycle of each member of $\mathcal{B}_{i}$ contains no vertex in $X_{i}$. By 4.2, it follows that the union of these jewel-boxes is a jewel-box in $G$, and so

$$
\sum_{1 \leq i \leq s}\left|Y_{i}\right| / 92928-\left|X_{i}\right| \leq \beta(G)
$$

But $\left|Y_{1}\right|+\cdots+\left|Y_{s}\right|=|V(G)|$, and $\left|X_{1}\right|+\cdots+\left|X_{s}\right|=2|E(S)| \leq 2|V(S)|$, so we deduce that $|V(G)| / 92928 \leq 2|V(S)|+\beta(G)$. Now every vertex of $S$ either belongs to the inner tree of $T$ or to $P$, and $G$ is 3-edge-colourable by the four-colour theorem; so by 4.8 and $4.4,|V(S)| \leq 90 \beta(G)$. Thus $|V(G)| / 92928 \leq 181 \beta(G)$, that is, $\beta(G) \geq|V(G)| / 16819968$. This proves 4.10 .

\section{The non-3-connected case.}

Now we extend 4.10 to all planar cubic graphs without cutedges. We begin with:

5.1 Let $G$ be a 3-edge-colourable 2-connected cubic graph. If $(T, \phi)$ is a 2-cut-decomposition of $G$ then $|V(T)| \leq 6 \beta(G)$.

Proof. Let $F_{1}, F_{2}, F_{3}$ be pairwise disjoint perfect matchings of $G$.

(1) Let $(T, \phi)$ be a 2-cut-decomposition of $G$ such that

- $\phi^{-1}(t) \neq \emptyset$ for each $t \in V(T)$

- $\phi^{-1}(f) \subseteq F_{3}$ for every edge $f \in E(T)$.

Then $|E(T)| \leq \beta(G)$.

Let $t \in V(T)$. Since $\phi^{-1}(f) \subseteq F_{3}$ for every edge $f \in E(T)$ incident with $t$, it follows that every edge $e=u v \in F_{1} \cup F_{2}$, if $\phi(u)=t$ then $\phi(v)=t$. Moreover, since $\phi^{-1}(t) \neq \emptyset$, it follows that there is an edge $e=u v \in F_{1} \cup F_{2}$ with $\phi(u)=t$. Consequently there is a cycle $C$ with $E(C) \subseteq F_{1} \cup F_{2}$ such that $V(C) \subseteq \phi^{-1}(t)$. Since this holds for each $t \in V(T)$, we deduce that there are at least $|V(T)|$ cycles within $F_{1} \cup F_{2}$, and so $|E(T)| \leq \beta(G)$ by 4.3 . This proves (1).

(2) For every 2-cut-decomposition $(T, \phi)$ of $G$, if $\phi^{-1}(t) \neq \emptyset$ for each $t \in V(T)$ then $|E(T)| \leq 3 \beta(G)$.

For let $f \in E(T)$, and let $e_{1}, e_{2}$ be the two edges in $\phi^{-1}(f)$. Since $\left\{e_{1}, e_{2}\right\}$ is a cut of even cardinality of a cubic graph, it follows that every perfect matching contains an even number of members of this cut, and in particular one of $F_{1}, F_{2}, F_{3}$ includes both of $e_{1}, e_{2}$. From the symmetry between 
$F_{1}, F_{2}, F_{3}$, we may assume that $\phi^{-1}(f) \subseteq F_{3}$ for at least one-third of all edges $f \in E(T)$, and so by contracting all other edges $f \in E(T)$, we obtain a 2-cut-decomposition $(S, \phi)$ with $|E(S)| \geq|E(T)| / 3$, satisfying the hypotheses of (1). Consequently $|E(S)| \leq \beta(G)$, and so $|E(T)| \leq 3 \beta(G)$. This proves $(2)$.

Now we deduce the theorem. Let $(T, \phi)$ be a 2-cut-decomposition of $G$, and let $n_{1}, n_{2}$ and $n_{3}$ be the number of vertices of $T$ of degree 1,2 and at least 3 respectively. From the definition of a cut-decomposition, $\phi^{-1}(t) \neq \emptyset$ for each $t \in V(T)$ with degree one or two; so by contracting edges of $T$ appropriately, we deduce that there is a 2-cut-decomposition $(S, \phi)$ of $G$ with $|V(S)| \geq n_{1}+n_{2}$, such that $\phi^{-1}(s) \neq \emptyset$ for each $s \in V(S)$. It follows from (2) that $|E(S)| \leq 3 \beta(G)$, and so $n_{1}+n_{2}-1 \leq$ $3 \beta(G)$. Since $T$ is a tree, it follows that $n_{3} \leq n_{1}-2$, and so

$$
|V(T)|=n_{1}+n_{2}+n_{3} \leq 2 n_{1}+n_{2}-2 \leq 2\left(n_{1}+n_{2}-1\right) \leq 6 \beta(G) .
$$

This proves 5.1 .

We would like to define a notion analogous to "3-hub" for a 2-cut-decomposition, but we have to be more careful. Let $(T, \phi)$ be a 2-cut-decomposition of $G$, and let $t \in V(T)$. We say that $t$ is solid if for every edge $f \in E(T)$ incident with $t$, and every $e \in \phi^{-1}(f), e$ is incident in $G$ with some vertex of $\phi^{-1}(t)$. Let $t \in V(T)$ be solid; let $X_{0}=\phi^{-1}(t)$, and let $f_{1}, \ldots, f_{k}$ be the edges of $T$ incident with $t$, where $f_{i}$ is incident with a vertex of $T_{i}$ for $1 \leq i \leq k$. Let $G^{\prime}$ be obtained from $G \mid X_{0}$ by adding a new edge $x_{i} y_{i}$ for $1 \leq i \leq k$, where $x_{i}, y_{i}$ are the two vertices in $X_{0}$ incident in $G$ with edges in $\phi^{-1}\left(f_{i}\right)$. We call $G^{\prime}$ the 2 - $h u b$ of $G$ at $t$. We need an analogue of 4.2 , as follows.

5.2 Let $(T, \phi)$ be a 2-cut-decomposition of a 2-connected cubic graph $G$, let $t_{1}, \ldots, t_{k} \in V(T)$ be solid, and for $1 \leq i \leq k$, let $H_{i}$ be the 2 -hub of $G$ at $t_{i}$. For $1 \leq i \leq k$ let $\mathcal{B}_{i}$ be a set of bracelets of $G$, such that

- $\mathcal{B}_{i}$ is a jewel-box of $H_{i}$, and

- if $C$ is the supporting cycle of a member of $\mathcal{B}_{i}$, then $V(C) \subseteq \phi^{-1}(t)$.

Then $\mathcal{B}_{1} \cup \cdots \cup \mathcal{B}_{k}$ is a jewel-box in $G$.

Proof. Suppose not. Let $w$ be the sum of the looks of a subset of $\mathcal{B}_{1} \cup \cdots \cup \mathcal{B}_{k}$, and for $i=-1,0,1$ let $F_{i}$ be the set of all $e \in E(G)$ with $w(e)=i$; and suppose there is a cut $D$ of $G$ such that every edge of $D$ belongs to $F_{-1}$ except for one, $f$ say, that belongs to $F_{0}$. Choose $D=\delta(X)$ and $f \in D$ with $|D|$ minimum, and, subject to that, with $|X|$ minimum; then, as in the proof of $4.2, G|X, G|(V(G) \backslash X)$ are both connected.

(1) Let $e \in T$, let $S_{1}, S_{2}$ be the two components of $T \backslash e$, and for $i=1,2$ let $Y_{i}=\phi^{-1}\left(V\left(S_{i}\right)\right)$. Then one of $X \cap Y_{1}, X \cap Y_{2}, Y_{1} \backslash X, Y_{2} \backslash X$ is empty.

Suppose all four of these sets are nonempty. Let $\delta\left(Y_{1}\right)=\left\{a_{1} a_{2}, b_{1} b_{2}\right\}$, where $a_{i}, b_{i} \in Y_{i}$ for $i=1,2$. Since $G|X, G|(V(G) \backslash X)$ are both connected, at least one of the edges $a_{1} a_{2}, b_{1} b_{2}$ has both ends in $X$, and at least one has both ends in $V(G) \backslash X$; so we may assume that $a_{1}, a_{2} \in X$ and $b_{1}, b_{2} \in V(G) \backslash X$. In particular, neither of $a_{1} a_{2}, b_{1} b_{2}$ belongs to $D$; so from the symmetry between $Y_{1}, Y_{2}$, we may assume that both ends of $f$ belong to $Y_{2}$. Now $\delta\left(Y_{1}\right) \subseteq F_{0}$, from the choice of $\mathcal{B}_{1}, \ldots, \mathcal{B}_{k}$, and so 
$a_{1} a_{2}, b_{1} b_{2} \in F_{0}$. Thus every edge of $\delta\left(X \cap Y_{1}\right)$ belongs to $F_{-1}$ except for exactly one in $F_{0}$, namely $a_{1} a_{2}$. From the minimality of $|D|$, it follows that $\delta\left(X \cap Y_{1}\right)|\geq| \delta(X) \mid$, and so $\left|\delta\left(X \cap Y_{2}\right)\right|=2$, and $\delta\left(X \cap Y_{1}\right)|=| \delta(X) \mid$, and yet $\left|X \cap Y_{1}\right|<|X|$, contrary to the minimality of $|X|$. This proves (1).

Since $G$ is 2-connected and therefore $|D| \geq 2$, we may choose $e \in D$ with $e \neq f$. Thus $\phi(e)=-1$, and so $e$ is an edge of the supporting cycle of some member of $\mathcal{B}_{1} \cup \cdots \cup \mathcal{B}_{k}$; say $e \in E\left(C_{1}\right)$ where $C_{1}$ is the supprting cycle of some $w_{1} \in \mathcal{B}_{1}$. Let $Y_{0}=\phi^{-1}\left(t_{1}\right)$. Let $e=x y$; then since $V\left(C_{1}\right) \subseteq Y_{0}$ it follows that $x, y \in Y_{0}$, and since $e \in \delta(X)$, we deduce that $X \cap Y_{0}, Y_{0} \backslash X$ are both nonempty. Let $S_{1}, \ldots, S_{s}$ be the components of $T \backslash\left\{t_{1}\right\}$, and for $1 \leq i \leq s$ let $e_{i}$ be the unique edge of $T$ incident with $t_{1}$ and an end in $V\left(S_{i}\right)$. For $1 \leq i \leq s$, let $Y_{i}=\phi^{-1}\left(S_{i}\right)$. Thus $Y_{0}, Y_{1}, \ldots, Y_{s}$ are pairwise disjoint subsets of $V(G)$ with union $V(G)$. The 2-hub $H_{1}$ of $G$ at $t_{1}$ is therefore obtained by contracting all edges of $G \mid Y_{i}$ and one of the two edges of $\phi^{-1}\left(e_{i}\right)$, for $1 \leq i \leq s$. Since $D$ is not a cut of $H_{1}$ (because $\mathcal{B}_{1}$ is a jewel-box of $\left.H_{1}\right)$ it follows that for some $i \in\{1, \ldots, s\}$, both $X \cap Y_{i}, Y_{i} \backslash X$ are nonempty. But since both $Y_{0} \cap X, Y_{0} \backslash X$ are nonempty, this contradicts (1) applied to the edge $e_{i}$. This proves 5.2.

Now we prove the main theorem of the paper, 2.2 , which we restate:

5.3 Let $G$ be a planar cubic graph with no cutedge. Then $\beta(G) \geq|V(G)| / 218659584$.

Proof. We proceed by induction on $|V(G)|$. If $G$ is not connected, the result follows from the inductive hypothesis applied to the components of $G$ (for the union of jewel-boxes in different components is a jewel-box in $G$, as is easily seen). Thus we may assume that $G$ is connected and hence 2 -connected, since it has no cutedge. If $G$ is 3 -connected the result follows from 4.10 , so we may assume that $G$ is not 3 -connected. Hence there is a 2-cut-decomposition $(T, \phi)$ of $G$; choose such a decomposition with $|V(T)|$ maximum. (This is possible by 5.1.)

(1) Let $t \in V(T)$ such that $\phi^{-1}(t) \neq \emptyset$. Then $t$ is solid, and the 2 -hub of $G$ at $t$ is 3 -edge-connected.

For let $f_{1}, \ldots, f_{k}$ be the edges of $T$ incident with $t$, and let $T_{1}, \ldots, T_{k}$ be the components of $T \backslash\{t\}$, where $f_{i}$ is incident with a vertex $t_{i}$ of $T_{i}$ for $1 \leq i \leq k$. Let $X_{0}=\phi^{-1}(t)$, and for $1 \leq i \leq k$ let $X_{i}=\phi^{-1}\left(T_{i}\right)$. To prove that $t$ is solid, let $e \in \phi^{-1}\left(f_{1}\right)$ say, where $e=u v$. Since $\phi^{-1}\left(f_{1}\right)=\delta\left(X_{1}\right)$, exactly one of $u, v \in X_{1}$, say $u$. We must show that $v \in X_{0}$. For suppose not; then $v$ is in one of $X_{2}, \ldots, X_{k}$, say $X_{2}$. Let $T^{\prime}$ be the tree obtained from $T$ by deleting the edges $f_{1}, f_{2}$ and adding a new vertex $s$ adjacent to $t_{1}, t_{2}, t$. Then $\left(T^{\prime}, \phi\right)$ is a 2-cut-decomposition of $G$ (to see this, note that $s$ has degree at least three in $G$, and every other vertex of $T$ has the same degree in $T$ and in $T^{\prime}$, except for $t$, and $\phi^{-1}(t) \neq \emptyset$.) But this contradicts the maximality of $|V(T)|$. This proves that $t$ is solid.

Let $H$ be the 2-hub of $G$ at $t$, and for $1 \leq i \leq k$ let $x_{i}, y_{i}$ be the two vertices in $X_{0}$ incident in $G$ with an edge of $\delta\left(X_{i}\right)$; thus $x_{i} y_{i}$ is an edge $e_{i}$ say of $H$. Suppose that $H$ is not 3-edge-connected; then there is a partition $Y_{1}, Y_{2}$ of $\phi^{-1}(t)$ into two nonempty subsets, such that there are exactly two edges of $H$ between $Y_{1}, Y_{2}$. Let $T^{\prime}$ be the tree obtained from $T$ by deleting $t$ and adding two new vertices $s_{1} s_{2}$, where $s_{1}, s_{2}$ are adjacent, and for $1 \leq i \leq k s_{1}$ is adjacent to $t_{i}$ if and only if $x_{i}, y_{i} \in Y_{1}$, and otherwise $s_{2}$ is adjacent to $t_{i}$. (In particular, if $e_{i}$ joins a vertex of $Y_{1}$ to a vertex of $Y_{2}$ then $s_{2}$ is adjacent to $t_{i}$.) Define $\phi^{\prime}: V(G) \rightarrow V\left(T^{\prime}\right)$ by: $\phi^{\prime}(v)=s_{i}$ if $v \in Y_{i}$ for $i=1,2$, and $\phi^{\prime}(v)=\phi(v)$ if $\phi(v) \neq t$. We claim that $\left(T^{\prime}, \phi^{\prime}\right)$ is 2-cut-decomposition of $G$. Note that $\phi^{\prime-1}\left(s_{i}\right) \neq \emptyset$ for $i=1,2$, so it remains to check that there are exactly two edges of $G$ in $\phi^{\prime-1}\left(s_{1} s_{2}\right)$. But since $t$ is solid, this 
set consists of all edges $u v$ of $G$ with $u \in Y_{1}$ and $v \in Y_{2}$ (which therefore belongs to $\delta_{H}\left(X_{1}\right)$ ) and all edges $u v$ of $G$ such that $u \in Y_{1}, v \in X_{i}$ and $e_{i} \in \delta_{H}\left(X_{1}\right)$. Since $\left|\delta_{H}\left(X_{1}\right)\right|=2$, it follows that there are exactly two edges in $\phi^{\prime-1}\left(s_{1} s_{2}\right)$. Thus $\left(T^{\prime}, \phi^{\prime}\right)$ is 2-cut-decomposition of $G$, contrary to the maximality of $|V(T)|$. This proves (1).

Let $t_{1}, \ldots, t_{k}$ be the vertices $t$ of $T$ such that $\phi^{-1}(t) \neq \emptyset$, and for $1 \leq i \leq k$ let $H_{i}$ be the 2-hub of $G$ at $t_{i}$. Let $Y_{i}$ be the set of edges $e=u v$ of $G$ such that $u, v \in \phi^{-1}\left(t_{i}\right)$, and let $X_{i}=E\left(H_{i}\right) \backslash Y_{i}$. Thus $\left|X_{i}\right|$ is the degree of $t_{i}$ in $T$. Let $n_{i}=\left|\phi^{-1}\left(t_{i}\right)\right|$. We claim there is a set $\mathcal{B}_{i}$ of bracelets of $G$, such that

- $\mathcal{B}_{i}$ is a jewel-box of $H_{i}$, and

- if $C$ is the supporting cycle of a member of $\mathcal{B}_{i}$, then $V(C) \subseteq \phi^{-1}(t)$

- $\left|\mathcal{B}_{i}\right| \geq n_{i} / 16819968-\left|X_{i}\right|$.

For if $n_{i} \leq 2$ and $X_{i} \neq \emptyset$, we may take $\mathcal{B}_{i}=\emptyset$, and if $n_{i} \leq 2$ and $X_{i}=\emptyset$ then $|V(G)| \leq 2$ and the result follows from 4.3. Thus we may assume that $n_{i} \geq 3$, and so $H_{i}$ is 3 -connected, since it is cubic and 3-edge-connected by (1). By 4.10 there is a jewel-box in $H_{i}$ of cardinality at least $n_{i} / 16819968$, and at most $\left|X_{i}\right|$ of its members have a supporting cycle that contains a member of $X_{i}$. Removing these members gives the jewel-box $\mathcal{B}_{i}$ as claimed. By 5.2, the union of these jewel-boxes is a jewel-box in $G$, and so

$$
\sum_{1 \leq i \leq k}\left(n_{i} / 16819968-\left|X_{i}\right|\right) \leq \beta(G) .
$$

But $n_{1}+\cdots+n_{k}=|V(G)|$, and

$$
\left|X_{1}\right|+\cdots+\left|X_{k}\right| \leq 2|E(T)| \leq 12 \beta(G)
$$

by 5.1 , so $|V(G)| / 16819968-12 \beta(G) \leq \beta(G)$, that is, $\beta(G) \geq|V(G)| / 218659584$. This proves 5.3 .

\section{References}

[1] T. Fowler, Unique Coloring of Planar Graphs, Ph.D. thesis, Georgia Institute of Technology, 1998.

[2] D. Kral, J.-S. Sereni and M. Stiebitz, "A new lower bound on the number of perfect matchings in cubic graphs", submitted for publication (manuscript 2008).

[3] L. Lovász and M. Plummer, Matching Theory, Annals of Discrete Math. 29, North Holland, Amsterdam, 1986.

[4] A. Schrijver, "Counting 1-factors in regular bipartite graphs", J. Combinatorial Theory, Ser. B 72 (1998), 122-135.

[5] H. Whitney, "A theorem on graphs", Ann. Math. 32 (1931), 378-390. 